\title{
ORIGINAL ARTICLE Pulmonary hypertension in preterm infants: results of a prospective screening program
}

\author{
CG Weismann ${ }^{1,2}$, JD Asnes $^{1}$, A Bazzy-Asaad $^{3}$, C Tolomeo $^{3}$, RA Ehrenkranz ${ }^{4}$ and MJ Bizzarro ${ }^{4}$
}

\begin{abstract}
OBJECTIVE: Determine prevalence and associations with pulmonary hypertension (PH) in preterm infants. STUDY DESIGN: Prospective institutional echocardiographic PH screening at 36 to 38 weeks' corrected gestational age (GA) for infants born < 32 weeks' GA who had bronchopulmonary dysplasia (BPD; group BPD), and infants without BPD who had a birth weight (BW) $<750 \mathrm{~g}$, or clinical suspicion for PH (group NoBPD).

RESULTS: Two hundred and four infants were screened (GA $25.9 \pm 2$ weeks, BW $831 \pm 286 \mathrm{~g}$ ). The PH prevalence in group BPD was higher than in group NoBPD $(44 / 159(28 \%)$ vs $5 / 45(11 \%) ; P=0.028)$. In group BPD, BW and GA were lower in infants with PH compared with NoPH. Following correction for BW and GA, necrotizing enterocolitis (NEC), severe intraventricular hemorrhage (IVH), atrial septal defect (ASD), and mortality were independently associated with PH in infants with BPD. In group NoBPD, NEC was the only identified factor associated with PH. Altogether, screening only those infants with NEC and infants with BPD who also had a BW $<840 \mathrm{~g}$ would have yielded a $84 \%$ sensitivity for detecting $\mathrm{PH}$, and reduced the number of screening echocardiograms by $43 \%$.

CONCLUSIONS: PH in prematurity is associated with NEC in infants with and without BPD. In infants with BPD, smaller GA and BW, severe IVH, ASD and mortality are also associated with $\mathrm{PH}$. Infants without identified $\mathrm{PH}$-associated factors may not require routine echocardiographic $\mathrm{PH}$ screening.
\end{abstract}

Journal of Perinatology (2017) 37, 572-577; doi:10.1038/jp.2016.255; published online 16 February 2017

\section{INTRODUCTION}

Over the past few decades, survival of premature infants has significantly increased. Bronchopulmonary dysplasia (BPD) is one of the most common morbidities associated with prematurity and with the development of pulmonary hypertension $(\mathrm{PH}) . \mathrm{PH}$ in this population is thought to be due to abnormal microvascular development and pulmonary vascular remodeling, resulting in reduced cross-sectional area of pulmonary vasculature. ${ }^{1}$ In addition, pulmonary vascular reactivity is increased rendering these infants more sensitive to periods of hypoxemia. ${ }^{2} \mathrm{PH}$ has been reported in 23 to $37 \%$ of premature infants in multiple retrospective studies with variable inclusion criteria, definitions for $\mathrm{PH}$, and echocardiographic interpretation. ${ }^{3-6}$ However, recent prospective studies suggest that the incidence of 'late' $\mathrm{PH}$, at $\sim 36$ weeks' corrected gestational age (CGA), may actually be lower (4 to $14 \%)^{7-9}$ Both prenatal and postnatal risk factors have been associated with $\mathrm{PH}$ in infants with BPD, including oligohydraminios, low 5-min APGAR score, lower birth weight (BW), small for GA, severe BPD, infection and patent ductus arteriosus (PDA). Outcomes associated with PH in infants with BPD include prolonged mechanical ventilation and need for prolonged supplemental oxygen therapy, increased length of hospital stay and, most importantly, increased mortality. ${ }^{3-7,10-12}$ To date, there are no published guidelines for PH screening in infants with BPD.

In 2010, we instituted a screening program in the Yale-New Haven Children's Hospital Neonatal Intensive Care Unit (NICU) for infants at perceived risk for PH (Figure 1). The aim of this study was to determine the prevalence of $\mathrm{PH}$ as well as associated risk factors for $\mathrm{PH}$ in a cohort of infants $<32$ weeks' gestation.

\begin{abstract}
METHODS
At Yale-New Haven Children's Hospital, we established an echocardiographic screening protocol for $\mathrm{PH}$ in preterm infants. We report data collected prospectively between April 2010 and November 2014. Screening echocardiograms were performed at 36 to 38 weeks' CGA. Inclusion criteria were (1) born $<32$ weeks' GA and diagnosed with BPD at 36 weeks' CGA (group BPD) or 2) born $<32$ weeks' GA and $<750 \mathrm{~g} \mathrm{BW}$ but without BPD (group NoBPD). In addition, screening was also performed at the request of the medical team for any infant $<32$ weeks' GA with pulmonary disease that did not meet criteria for BPD at 36 weeks' CGA but had a clinical suspicion for PH (group NoBPD).

We collected data on maternal and perinatal factors, neonatal demographics, major neonatal morbidities and outcomes for inclusion in our analyses. Maternal factors included the presence of chorioamnionitis, maternal hypertension and administration of antenatal steroids. Neonatal factors included gender, GA, BW and small for GA status. Neonatal morbidity data included BPD, early- and late-onset sepsis, necrotizing enterocolitis (NEC), intraventricular hemorrhage (IVH) and presence of a PDA. BPD was graded by severity as mild, moderate or severe using the consensus definition by National Institute of Child Health and Human Development (2000). ${ }^{13}$ Early-onset sepsis was defined as a positive blood culture(s) obtained at $\leqslant 72 \mathrm{~h}$ of life, which yielded a traditional neonatal pathogen (e.g. Escherichia coli, Group B streptococcus). Late-onset sepsis was defined as a positive blood culture(s) obtained at $>72 \mathrm{~h}$ of life which yielded a traditional neonatal pathogen or a commensal species (e.g. Staphylococcus epidermidis). ${ }^{14}$ Blood cultures that yielded
\end{abstract}

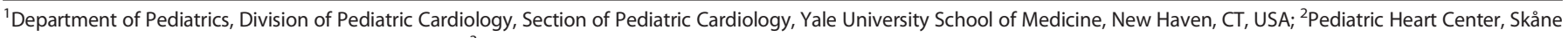

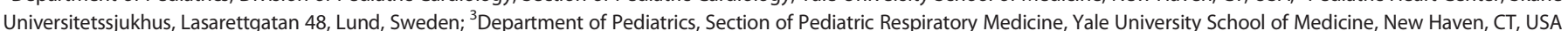

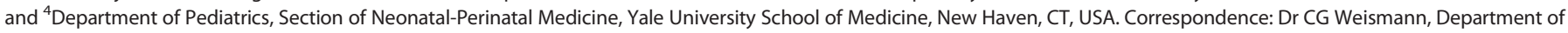
Pediatrics, Division of Pediatric Cardiology, Section of Pediatric Cardiology, Yale University School of Medicine, 333 Cedar Street, LLCI 302, New Haven, CT 06510, USA

E-mail: constance.weismann@yale.edu

Received 22 February 2016; revised 2 November 2016; accepted 13 December 2016; published online 16 February 2017 
coagulase-negative staphylococci were reviewed using specific criteria from the Centers for Disease Control and Prevention. ${ }^{15}$ NEC was defined according to the modified Bell's staging and included only those $\geqslant$ stage IIA. ${ }^{16}$ Surgical NEC included only those cases that went on to require surgical intervention. IVH was graded based on the classification system developed by Papile et al. ${ }^{17}$ with severe IVH including only those of grade III or IV. PDA included only those deemed to be hemodynamically significant and was diagnosed via echocardiogram as reviewed by a pediatric cardiologist. Last, outcome data included death, cumulative time (in days) requiring mechanical ventilation through an endotracheal tube, length of hospital stay, CGA at the time of discharge and whether or not an infant was discharged from the NICU on supplemental oxygen.

A screening echocardiogram was conducted at 36 to 38 weeks' CGA using the Philips IE33 (Philips Medical Systems, Andover, MA, USA) or Siemens Sequoia SC2000 (Siemens Medical Solutions USA, Mountain View, CA, USA) equipment. All echocardiograms were performed by a pediatric

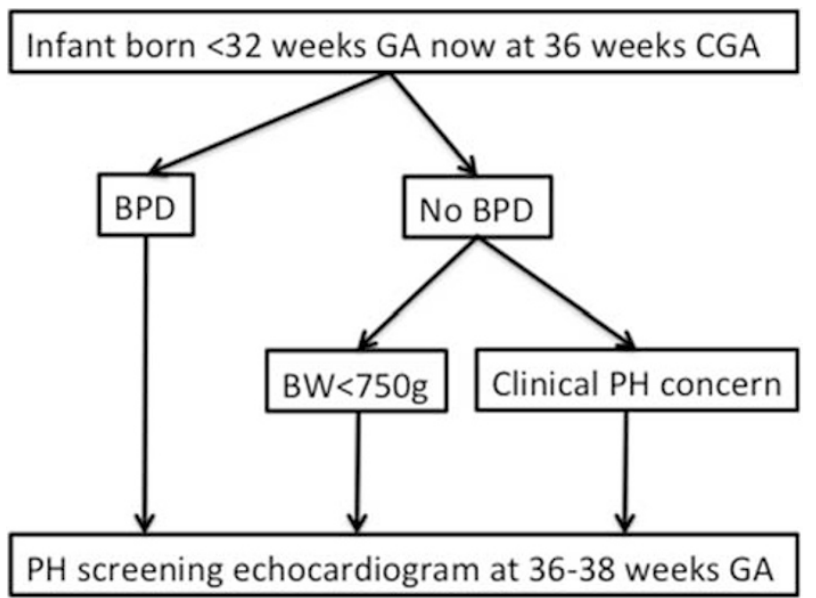

Figure 1. Echocardiographic screening algorithm for pulmonary hypertension $(\mathrm{PH})$ in preterm infants that was used for this study. BPD, bronchopulmonary dysplasia; BW, body weight; CGA, corrected gestational age. echocardiography technician using our institutional PH protocol. Echocardiograms were interpreted by a single pediatric echocardiographer (CGW) who was blinded to the clinical status of the patient. PH was defined as a tricuspid regurgitation jet gradient of $>36 \mathrm{~mm} \mathrm{Hg}$ or presence of septal flattening in systole. We also documented presence of a small or greater atrial septal defect (ASD) or PDA. A patent foramen ovale was defined as the space between a well-developed septum primum and a normally formed septum secundum, and was considered normal. ${ }^{18} \mathrm{~A}$ secundum ASD was defined by the presence of deficiency of the septum primum.

Infants with evidence of $\mathrm{PH}$ underwent follow-up echocardiograms at individualized intervals. We documented the presence or absence of $\mathrm{PH}$ at the time of last follow-up but no later than 1 year of age. Infants who had a normal PH screening echocardiogram at 36 weeks' CGA and who were under the care of the Pediatric Respiratory Medicine service underwent repeat echocardiography at $\sim 1$ year of age.

For statistical analyses continuous variables were expressed as mean (s.d.), unless otherwise indicated. Categorical variables were expressed as numbers (frequencies). For comparisons between groups, the $t$-test for independent samples was used for continuous data. Fisher's exact or $X^{2}$ tests were used as appropriate for comparison of categorical variables between groups. Binary logistic regression was used to correct for differences in BW and GA between the groups and adjusted odds ratios (ORs) with 95\% confidence intervals (Cls) were determined. Receiver operating characteristics curves were created. The set significance level was $P<0.05$. For multiple comparisons, the Bonferroni correction was used (e.g. NEC vs surgical NEC, $P<0.025$ was considered significant). Statistical analyses were performed using Statistical Package for Social Sciences, version 21 (IBM SPSS, Chicago, IL, USA).

\section{RESULTS}

Three hundred and ninety-eight infants born at $<32$ weeks' GA and still in our NICU at 36 weeks' CGA were eligible for inclusion (Figure 2). One hundred and eighty-nine infants were excluded because they did not meet the inclusion criteria (i.e. they were $<32$ weeks' GA, $\geqslant 750 \mathrm{~g}$ BW but without BPD or clinical suspicion for $\mathrm{PH}$ ). We excluded an additional two infants with congenital heart disease and associated elevated right heart pressure. Three infants with BPD never had a screening echocardiogram before discharge (Figure 2). Two hundred and four infants $<32$ weeks'

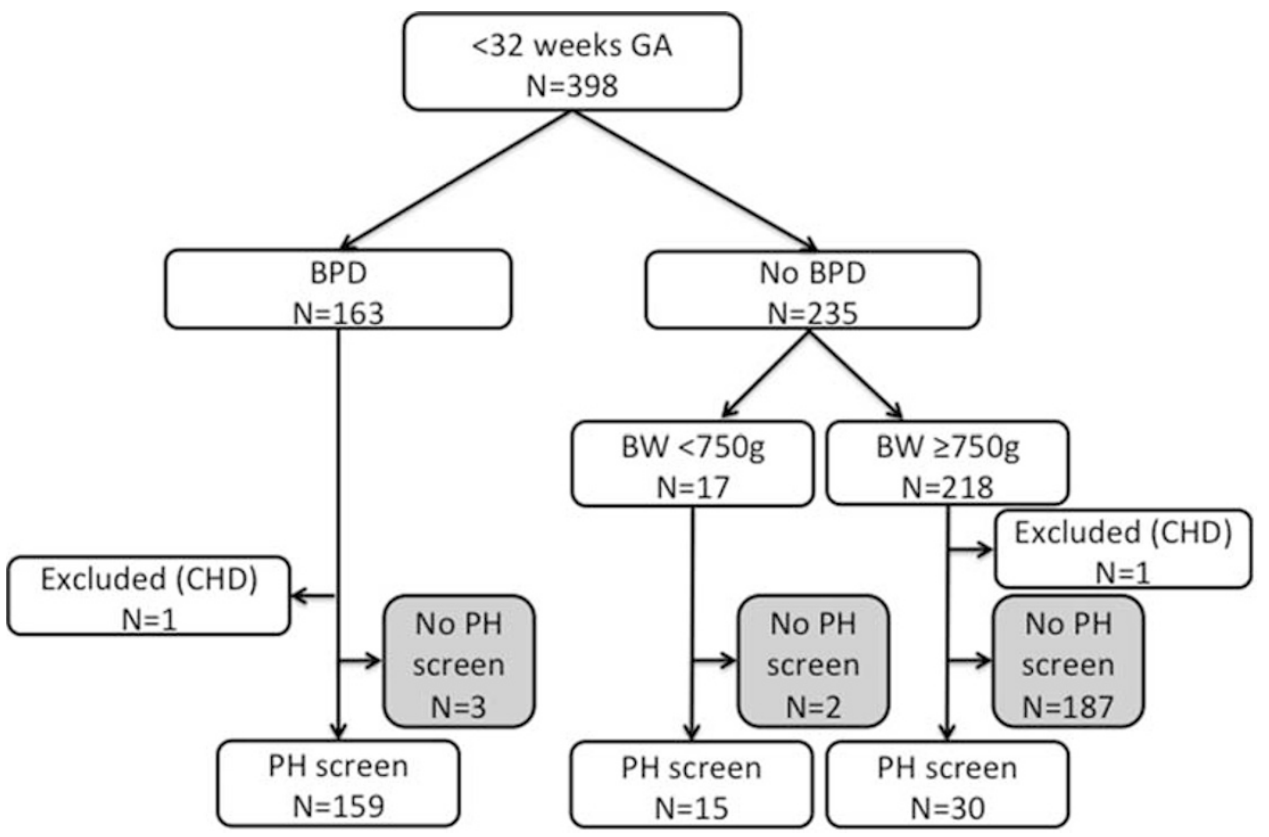

Figure 2. Consort diagram showing all infants born $<32$ weeks' gestational age (GA) who were hospitalized in the Yale-New Haven Children's Hospital neonatal intensive care unit at 36 weeks corrected GA (CGA). Eligible infants who did not have a pulmonary hypertension (PH) screening echocardiogram (gray). BPD, bronchopulmonary dysplasia; CHD, coronary heart disease. 
Table 1. Characteristics of groups BPD and NoBPD, each stratified by subgroups PH and NoPH

\begin{tabular}{|c|c|c|c|c|c|c|}
\hline \multirow[t]{2}{*}{ Variable } & \multicolumn{3}{|c|}{ Group BPD } & \multicolumn{3}{|c|}{ Group NoBPD } \\
\hline & $P H(\mathrm{n}=44)$ & $\mathrm{NoPH}(\mathrm{N}=115)$ & P-value & $P H(\mathrm{n}=5)$ & $\mathrm{NoPH}(\mathrm{N}=40)$ & P-value \\
\hline \multicolumn{7}{|l|}{ Maternal factors } \\
\hline Chorioamnionitis & $18(41 \%)$ & $54(47 \%)$ & 0.482 & $3(60 \%)$ & 15 (38\%) & 0.375 \\
\hline Maternal hypertension & $12(27 \%)$ & $24(21 \%)$ & 0.403 & $0(0 \%)$ & $11(28 \%)$ & 0.313 \\
\hline Antenatal steroids & 39 (93\%) & $106(94 \%)$ & 1 & $5(100 \%)$ & 39 (98\%) & 1 \\
\hline Gestational age (weeks) & $25.0(1.7)$ & $25.8(1.9)$ & 0.023 & $27(1.9)$ & $27.4(2.0)$ & 0.684 \\
\hline Birth weight (g) & $703(204)$ & $824(257)$ & 0.006 & $930(242)$ & $979(370)$ & 0.775 \\
\hline Small for gestational age & $9(21 \%)$ & $21(18 \%)$ & 0.821 & $0(0 \%)$ & $12(30 \%)$ & 0.303 \\
\hline \multicolumn{7}{|l|}{ Postnatal factors } \\
\hline BPD grade & & & 0.365 & & & \\
\hline Severe IVH (grade III/IV) & $12(27 \%)$ & $9(8 \%)$ & 0.003 & $0(0 \%)$ & $1(3 \%)$ & 1 \\
\hline PDA & 17 (39\%) & 37 (32\%) & 0.459 & $2(40 \%)$ & $3(8 \%)$ & 0.087 \\
\hline PDA ligation & $11(25 \%)$ & $21(18 \%)$ & 0.379 & $0(0 \%)$ & $2(5 \%)$ & 1 \\
\hline NEC ( $\geqslant$ stage $\| A)$ & $13(30 \%)$ & $8(7 \%)$ & $<0.001$ & $3(60 \%)$ & $4(10 \%)$ & 0.021 \\
\hline Surgical NEC & $8(18 \%)$ & $5(4 \%)$ & 0.008 & $2(40 \%)$ & $3(8 \%)$ & 0.087 \\
\hline \multicolumn{7}{|l|}{ Outcomes } \\
\hline Death & $6(14 \%)$ & $3(3 \%)$ & 0.014 & $0(0 \%)$ & $0(0 \%)$ & $\mathrm{N} / \mathrm{A}$ \\
\hline Time on ventilator (days) & $63(63)$ & $55(66)$ & 0.487 & $26(17)$ & $13(17)$ & 0.105 \\
\hline Length of stay (days) of patients discharged alive & $153(61)$ & $135(70)$ & 0.178 & $96(21)$ & $98(51)$ & 0.949 \\
\hline CGA at discharge/transfer & 47 (9) & $45(10)$ & 0.314 & $41(3)$ & $41(7)$ & 0.859 \\
\hline Discharge/transfer on oxygen & $21(48 \%)$ & $42(37 \%)$ & 0.209 & $0(0 \%)$ & $0(0 \%)$ & $\mathrm{N} / \mathrm{A}$ \\
\hline \multicolumn{7}{|l|}{ Screening echocardiogram at 36-38 weeks GA } \\
\hline
\end{tabular}

gestation who met the inclusion criteria and underwent a $\mathrm{PH}$ screening echocardiogram at 36 to 38 weeks' CGA at Yale-New Haven Children's Hospital were included in this study. Group BPD included 159 infants diagnosed with BPD at 36 weeks' CGA. ${ }^{13}$ Group NoBPD included 45 infants born at $<32$ weeks' GA. These included 15 infants screening strictly for BW $<750 \mathrm{~g}$ as well as 30 infants with non-BPD pulmonary disease and a clinical suspicion for $\mathrm{PH}(n=30)$.

\section{Group BPD}

In group BPD, 44 of 159 infants (27.7\%) had echocardiographic evidence of $\mathrm{PH}$ at the time of the screening echocardiogram. Of those, 35 had systolic septal flattening only, 1 had a tricuspid regurgitation jet gradient $>36 \mathrm{~mm} \mathrm{Hg}$ only and 8 met both the criteria. Infants with PH were of lower birth BW and GA, and were more likely to have early-onset sepsis, severe IVH and NEC (medical and surgical) as compared with those without $\mathrm{PH}$ (Table 1). At the time of the $\mathrm{PH}$ screening echocardiogram, infants with PH were also more likely to have an ASD (Table 1). Left-right interatrial shunting was present in all but of the two infants. The presence of an ASD was strongly associated with right ventricular hypertrophy (ASD 16/32 (50\%) vs no ASD 21/127 (17\%), $P<0.001$ ) and dilation (ASD 16/32 (50\%) vs no ASD 8/127 (6\%), $P<0.001$ ). No association between the severity of BPD and the prevalence of $\mathrm{PH}$ was determined. Following correction for $\mathrm{GA}$ and $\mathrm{BW}$ in a logistic regression model, all NEC, surgical NEC, severe IVH and the presence of an ASD remained independent predictors of $\mathrm{PH}$ in the cohort with BPD (Table 2). Early-onset sepsis occurred only in infants with $\mathrm{PH}$ and therefore could not be used in the logistic regression model. $\mathrm{PH}$ was also determined to be an independent predictor for death before discharge from the NICU (Tables 1 and 2). In infants with BPD, length of stay, duration of mechanical ventilation and discharge/transfer on oxygen did not differ between infants with and without PH (Table 1).

Two infants in group BPD underwent a diagnostic cardiac catheterization, 5 infants were placed on $\mathrm{PH}$-specific oral agents, 10 received inhaled nitric oxide (NO), and 3 received both oral and inhaled treatment. Thirty-five of $44(80 \%)$ infants in the $\mathrm{PH}$ subgroup underwent follow-up echocardiograms. At the time of 
Table 2. ORs (unadjusted and adjusted for birth weight and gestational age) for factors associated with PH within the BPD group

\begin{tabular}{lcccc}
\hline Variable & $\begin{array}{c}\text { Unadjusted OR } \\
(95 \% \mathrm{Cl})\end{array}$ & P-value & $\begin{array}{c}\text { Adjusted OR } \\
(95 \% \mathrm{Cl})\end{array}$ & P-value \\
\hline $\begin{array}{l}\text { Gestational age } \\
\text { (weeks) }\end{array}$ & $0.8(0.64-0.97)$ & 0.026 & & \\
Birth weight (g) & 0.997 & 0.008 & & \\
& $(0.996-0.999)$ & & & \\
NEC & $5.6(2.1-14.8)$ & $<0.001$ & $5.5(1.9-15.4)$ & 0.001 \\
Surgical NEC & $4.7(1.6-14.1)$ & 0.006 & $4.0(1.3-12.5)$ & 0.018 \\
Severe IVH & $4.4(1.7-11.4)$ & 0.002 & $3.7(1.3-10.4)$ & 0.013 \\
ASD & $3.5(1.6-7.9)$ & 0.002 & $3.1(1.4-7.1)$ & 0.007 \\
Death & $5.9(1.4-25)$ & 0.015 & $5.3(1.2-23.8)$ & 0.031 \\
\hline
\end{tabular}

Abbreviations: ASD, atrial septal defect; BPD, bronchopulmonary dysplasia; $\mathrm{Cl}$, confidence interval; $\mathrm{IVH}$, intraventricular hemorrhage; N/A, not applicable; NEC, necrotizing enterocolitis; OR, odds ratio. Data are presented as OR $(95 \% \mathrm{Cl})$.

their most recent follow-up, only six (17\%) still had evidence of $\mathrm{PH}$ (Table 1). Fifty-nine of 115 infants with BPD but without initial evidence of $\mathrm{PH}$ had follow-up echocardiograms. Only one infant had newly developed $\mathrm{PH}$ at the time of a clinical deterioration. The infant died secondary to NEC totalis.

\section{Group NoBPD}

In the NoBPD group, 5 of $45(11 \%)$ infants had echocardiographic evidence of $\mathrm{PH}$. There were no significant differences in select maternal factors, gender, BW or GA between infants with or without $\mathrm{PH}$ in this group. The only postnatal factor associated with $\mathrm{PH}$ in the NoBPD group was NEC (Table 1). There were no differences in echocardiographic and/or outcome variables between infants with or without $\mathrm{PH}$ in this cohort, and no infants in this group died before NICU discharge (Table 1). Of note, there was no difference in the prevalence of PH at 36 to 38 weeks' CGA when comparing the subgroup of infants with $\mathrm{BW}<750 \mathrm{~g}$ with those with $\mathrm{BW} \geqslant 750 \mathrm{~g}(2 / 15(13 \%)$ vs $3 / 30(10 \%) ; P=1)$. At the time of the most recent follow-up, none of the 25 infants with available echocardiographic data had evidence of $\mathrm{PH}$ (Table 1).

\section{Group BPD and NoBPD combined}

In both groups BPD and NoBPD alike, NEC was the most significant factor associated with $\mathrm{PH}$, with $57 \%(16 / 28)$ having echocardiographic evidence of $\mathrm{PH}$ at 36 to 38 weeks' CGA. The prevalence of $\mathrm{PH}$ for infants who did not have a history of NEC was $22 \%(31 / 138)$ for group BPD compared with $5 \%(2 / 38)$ for group NoBPD $(P=0.018)$. In a subgroup analysis of group BPD without NEC, lower BW (area under the curve $0.619,95 \% \mathrm{Cl}: 0.504$ to 0.734 , $P=0.044$ ) was associated with $\mathrm{PH}$, whereas $\mathrm{GA}$ (area under the curve $0.568,95 \% \mathrm{Cl}: 0.0 .456$ to $0.679, P=0.253)$ and severe IVH (PH in severe IVH $5 / 13(38 \%)$ vs $26 / 125(21 \%)$ if no severe IVH; $P=0.167$ ) were not. Based on these results, a BW $<840 \mathrm{~g}$ as a predictor of $\mathrm{PH}$ was associated with a $80 \%$ sensitivity and $41 \%$

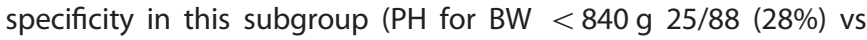
$6 / 50(12 \%)$ for $B W \geqslant 840 \mathrm{~g} ; P=0.033)$.

Therefore, if we limited $\mathrm{PH}$ screen to only those infants with NEC, and to those with BPD who also had a BW $<840 \mathrm{~g}$, the sensitivity for detecting $\mathrm{PH}$ would have been $84 \%$. This approach would have missed eight infants with echocardiographic evidence of $\mathrm{PH}$. However, by applying these more restrictive criteria for $\mathrm{PH}$ screening, we would have reduced the number of echocardiograms performed by 88 (i.e. by $43 \%$ ).

\section{DISCUSSION}

This is the first investigation of PH in infants $<32$ weeks' GA with BPD that prospectively identified NEC (both medical and surgical), surgical NEC, severe IVH, the presence of an ASD and mortality as factors independently associated with $\mathrm{PH}$ at 36 to 38 weeks' CGA. In infants < 32 weeks' GA and without BPD, NEC was the only identifiable factor associated with $\mathrm{PH}$.

The prevalence of $\mathrm{PH}$ in our study is within the previously reported range for $\mathrm{PH}$ in prematurity of up to $36 \% .^{3-6}$ However, two recent prospective studies suggest that the incidence of 'late' $\mathrm{PH}$, at $\sim 36$ weeks' CGA, may actually be lower (4 to $14 \%)^{8,9}$ This wide range may be attributed to the retrospective design of most studies, lack of prospective screening protocols, variability in inclusion criteria, time points when the echocardiograms were performed, classification of $\mathrm{PH}$ based on the clinical interpretation of echocardiograms by different readers and variable echocardiographic definitions for $\mathrm{PH}$.

The gold standard for diagnosing $\mathrm{PH}$ is a mean pulmonary arterial pressure of $>25 \mathrm{~mm} \mathrm{Hg}$ via cardiac catheterization. ${ }^{19}$ Interestingly, this definition applies to adults, children and infants alike, even though there are significant differences in systemic arterial blood pressure from infancy to adulthood. Using echocardiography as a screening tool for $\mathrm{PH}$, an estimated systolic pulmonary arterial pressure $\geqslant 35$ to $40 \mathrm{~mm} \mathrm{Hg}$ or the presence of end-systolic septal flattening are considered abnormal. ${ }^{9,20,21}$ Echocardiographic presence of end-systolic septal flattening is generally thought to indicate more than half systemic right ventricular systolic pressure. This was first described over 30 years ago in a study of 26 children who underwent cardiac catheterization and echocardiography simultaneously. ${ }^{20}$ Assessment of septal flattening, however, has significant interobserver variability. ${ }^{22}$ Quantitative estimates of septal flattening may therefore be useful in the absence of enough tricuspid regurgitation to estimate right ventricular systolic pressure. ${ }^{22-25}$ The left ventricular end-systolic eccentricity index (SEI) quantifies the degree of septal flattening. A SEI of 1 would be considered normal, septal flattening is recognized by a single observer at an SEI $\geqslant 1.15$, but greater than half systemic right ventricular pressure estimates was only noted at SEI $\geqslant 1.3$. $^{22}$ In our patient cohort, changing the definition of $\mathrm{PH}$ to $\mathrm{SEI} \geqslant 1.3$ would decrease the prevalence of $\mathrm{PH}$ from 27 to $10 \%$. Although SEl as a quantitative measure of septal flattening may increase reproducibility, correlation to actual hemodynamic data will remain challenging until simultaneous cardiac catheterization is performed as part of a larger multicenter study.

BPD has been previously reported to be a major independent risk factor for $\mathrm{PH}$, especially if severe. ${ }^{3,5,8,9}$ While BPD was associated with $\mathrm{PH}$ in our study, severity was not. This discrepancy may be due to variable definitions of BPD. While we used $\mathrm{NIH}$ criteria, we did not perform physiologic testing. ${ }^{7,9}$

In our study, NEC (medical and surgical NEC combined as well as surgical NEC alone) and severe IVH remained independently associated factors for PH in infant with BPD after controlling for GA and BW. In prior investigations, sepsis, inflammation and hypoxemia have all been associated with acute and/or chronic development of $\mathrm{PH}^{26,27}$ Diminished endothelial NO production by endothelial NO synthase can lead to PH in those clinical settings, which has also been described in infants with NEC. ${ }^{28}$ In addition, there is some evidence linking insufficient supply of endogenous $\mathrm{NO}$ in premature infants to BPD and IVH. ${ }^{29}$ This may explain why $\mathrm{NEC}$ and severe IVH were identified as $\mathrm{PH}$-associated factors in our cohort of preterm infants.

Risk stratification of preterm infants may enable us to reduce the number of screening echocardiograms performed. Biochemical markers may complement echocardiographic PH screening. $\mathrm{N}$-terminal pro-B-type natriuretic peptide is a potential biochemical marker that is used clinically for monitoring patients with $\mathrm{PH}$. 
Recently, N-terminal pro-B-type natriuretic peptide was found to be consistently elevated $>1000 \mathrm{pg} \mathrm{ml}^{-1}$ in preterm infants with BPD and PH at 36 to 38 weeks' CGA, when compared with controls. $^{30}$ If those results are validated in a larger study, $\mathrm{N}$-terminal pro-B-type natriuretic peptide may replace echocardiography as the primary screening method for infants deemed to be at low risk for $\mathrm{PH}$.

Prematurely born infants have a higher prevalence of congenital heart disease. ${ }^{31}$ In our investigation, infants with BPD and $\mathrm{PH}$ at the time of the screening echocardiogram were significantly more likely to have an ASD. It has been suggested that increased pulmonary blood flow may further enhance the pulmonary vascular remodeling already induced by other factors and result in pulmonary vascular disease. ${ }^{32}$ Early ASD closure during infancy with symptomatic improvement has been reported, and should be considered in preterm infants with BPD and $\mathrm{PH} .{ }^{33}$

Our overall mortality rate for infants with $\mathrm{PH}$ was $12 \%$, which is at the lower end of published associated mortality rates of 10 to $38 \%$. $3,5,9,10,21$ Length of stay, duration of mechanical ventilation and discharge or transfer on oxygen did not differ between infants with and without $\mathrm{PH}$ in our cohort. Prior investigations have reported conflicting data regarding differences in these outcomes between infants with and without $\mathrm{PH}^{3,5,9,21}$ In our NICU, once $\mathrm{PH}$ was identified medical management was altered to avoid hypercarbia and to allow for higher oxygen saturations. If $\mathrm{PH}$ remained significant and persistent, we considered diagnostic cardiac catheterization before starting anti-pulmonary hypertensive agents. In those infants too unstable to undergo cardiac catheterization, inhaled $\mathrm{NO}$ and/or Sildenafil were empirically initiated. Overall, only a small number of infants received $\mathrm{PH}$ specific therapy. Prospective screening for $\mathrm{PH}$ in at-risk infants allowed us to adjust medical management in an effort to optimize cardiopulmonary hemodynamics. We speculate that early recognition of $\mathrm{PH}$ may improve outcome.

The strengths of this study are the prospective design of our screening program, and the interpretation of echocardiograms by a single reader (CGW) who was blinded to the patient's clinical status. This study also has several limitations, including the possibility of a type II error given the relatively small number of infants with $\mathrm{PH}$, especially in the NoBPD group. Infants without BPD who had a BW $>750 \mathrm{~g}$ were only screened for $\mathrm{PH}$ at 36 to 38 weeks' CGA if there was a clinical concern allowing for selection bias. Further, follow-up echocardiograms to evaluate for new or persistent PH beyond 36 weeks' PMA were performed at variable time points, but usually at $\sim 1$ year of age if no clinical concerns existed to warrant sooner evaluation. EKG as a screening test was not part of this screening algorithm. Additionally, the gold standard for diagnosing $\mathrm{PH}$ is cardiac catheterization, an invasive test that was only performed in selected patients with echocardiograms suggesting significant $\mathrm{PH}$ at more than one time point. Last, given the highly variable prevalence of prematurity-related comorbidities and respiratory management, our findings may not be generalizable and should be evaluated in a larger multicenter study.

\section{CONCLUSION}

In a cohort of premature infants with BPD and after controlling for GA and BW, NEC, severe IVH, the presence of an ASD and mortality were identified as independent factors associated with $\mathrm{PH}$ at 36 to 38 weeks' CGA. NEC was the only factor associated with PH in preterm infants without BPD. The variability in reported risk factors for $\mathrm{PH}$ in premature infants underscores the complexity of this disease process in this population. Our results need to be validated in a larger multicenter study to suggest an echocardiographic PH screening algorithm for preterm infants that can be applied across multiple institutions. A better understanding of demographic and risk factors associated with $\mathrm{PH}$ may reduce the total number of screening echocardiograms performed and, with it, the associated costs.

\section{CONFLICT OF INTEREST}

The authors declare no conflict of interest.

\section{ACKNOWLEDGEMENTS}

This work was supported by the Department of Pediatrics, Yale University School of Medicine.

\section{REFERENCES}

1 Ambalavanan N, Mourani P. Pulmonary hypertension in bronchopulmonary dysplasia. Birth Defects Res A 2014; 100(3): 240-246.

2 Mourani PM, Ivy DD, Gao D, Abman SH. Pulmonary vascular effects of inhaled nitric oxide and oxygen tension in bronchopulmonary dysplasia. Am J Respir Crit Care Med 2004; 170(9): 1006-1013.

3 An HS, Bae EJ, Kim GB, Kwon BS, Beak JS, Kim EK et al. Pulmonary hypertension in preterm infants with bronchopulmonary dysplasia. Korean Circ J 2010; 40(3): 131-136.

4 Ali Z, Schmidt P, Dodd J, Jeppesen DL. Predictors of bronchopulmonary dysplasia and pulmonary hypertension in newborn children. Dan Med J 2013; 60 (8): A4688.

5 Slaughter JL, Pakrashi T, Jones DE, South AP, Shah TA. Echocardiographic detection of pulmonary hypertension in extremely low birth weight infants with bronchopulmonary dysplasia requiring prolonged positive pressure ventilation. J Perinatol 2011; 31(10): 635-640.

$6 \mathrm{Kim}$ GB. Pulmonary hypertension in infants with bronchopulmonary dysplasia. Korean J Pediatr 2010; 53(6): 688-693.

7 Bhat R, Salas AA, Foster C, Carlo WA, Ambalavanan N. Prospective analysis of pulmonary hypertension in extremely low birth weight infants. Pediatrics 2012; 129(3): e682-e689.

8 Mirza H, Ziegler J, Ford S, Padbury J, Tucker R, Laptook A. Pulmonary hypertension in preterm infants: prevalence and association with bronchopulmonary dysplasia. J Pediatr 2014; 165(5): 909-914 e901.

9 Mourani PM, Sontag MK, Younoszai A, Miller JI, Kinsella JP, Baker CD et al. Early pulmonary vascular disease in preterm infants at risk for bronchopulmonary dysplasia. Am J Respir Crit Care Med 2015; 191(1): 87-95.

10 Khemani E, McElhinney DB, Rhein L, Andrade O, Lacro RV, Thomas KC et al. Pulmonary artery hypertension in formerly premature infants with bronchopulmonary dysplasia: clinical features and outcomes in the surfactant era. Pediatrics 2007; 120(6): 1260-1269.

11 Stuart BD, Sekar P, Coulson JD, Choi SE, McGrath-Morrow SA, Collaco JM. Healthcare utilization and respiratory morbidities in preterm infants with pulmonary hypertension. J Perinatol 2013; 33(7): 543-547.

12 Check J, Gotteiner N, Liu X, Su E, Porta N, Steinhorn R et al. Fetal growth restriction and pulmonary hypertension in premature infants with bronchopulmonary dysplasia. J Perinatol 2013; 33(7): 553-557.

13 Ehrenkranz RA, Walsh MC, Vohr BR, Jobe AH, Wright LL, Fanaroff AA et al. Validation of the National Institutes of Health consensus definition of bronchopulmonary dysplasia. Pediatrics 2005; 116(6): 1353-1360.

14 Bizzarro MJ, Raskind C, Baltimore RS, Gallagher PG. Seventy-five years of neonatal sepsis at Yale: 1928-2003. Pediatrics 2005; 116(3): 595-602.

15 Garner JS, Jarvis WR, Emori TG, Horan TC, Hughes JM. CDC definitions for nosocomial infections, 1988. Am J Infect Control 1988; 16(3): 128-140.

16 Kliegman RM, Walsh MC. Neonatal necrotizing enterocolitis: pathogenesis, classification, and spectrum of illness. Curr Probl Pediatr 1987; 17(4): 213-288.

17 Papile LA, Burstein J, Burstein R, Koffler H. Incidence and evolution of subependymal and intraventricular hemorrhage: a study of infants with birth weights less than 1,500 gm. J Pediatr 1978; 92(4): 529-534.

18 Lai WW, Mertens LL, Cohen MS, Geva T. Echocardiography in Pediatric and Congenital Heart Disease. Blackwell Publishing: Hoboken, NJ, USA, 2009.

19 Hoeper MM, Bogaard HJ, Condliffe R, Frantz R, Khanna D, Kurzyna M et al. Definitions and diagnosis of pulmonary hypertension. J Am Coll Cardiol 2013; 62(25 Suppl): D42-D50.

20 King ME, Braun H, Goldblatt A, Liberthson R, Weyman AE. Interventricular septal configuration as a predictor of right ventricular systolic hypertension in children: a cross-sectional echocardiographic study. Circulation 1983; 68(1): 68-75.

$21 \mathrm{Kim}$ DH, Kim HS, Choi CW, Kim EK, Kim BI, Choi JH. Risk factors for pulmonary artery hypertension in preterm infants with moderate or severe bronchopulmonary dysplasia. Neonatology 2012; 101(1): 40-46. 
22 Abraham S, Weismann CG. Left ventricular end-systolic eccentricity index for assessment of pulmonary hypertension in infants. Echocardiography 2016; 33: 910-915.

23 D'Alto M, Romeo E, Argiento P, Pavelescu A, Melot C, D'Andrea A et al. Echocardiographic prediction of pre- versus postcapillary pulmonary hypertension. J Am Soc Echocardiogr 2015; 28(1): 108-115.

24 Haddad F, Guihaire J, Skhiri M, Denault AY, Mercier O, Al-Halabi S et al. Septal curvature is marker of hemodynamic, anatomical, and electromechanical ventricular interdependence in patients with pulmonary arterial hypertension. Echocardiography 2014; 31(6): 699-707.

25 Lopez-Candales A. Determinants of an abnormal septal curvature in chronic pulmonary hypertension. Echocardiography 2015; 32(1): 49-55.

26 Albertini M, Ciminaghi B, Mazzola S, Clement MG. Improvement of respiratory function by bosentan during endotoxic shock in the pig. Prostaglandins Leukot Essent Fatty Acids 2001; 65(2): 103-108.

27 Toney BM, Fisher AJ, Albrecht M, Lockett AD, Presson RG, Petrache I et al. Selective endothelin-A receptor blockade attenuates endotoxin-induced pulmonary hypertension and pulmonary vascular dysfunction. Pulm Circ 2014; 4(2): 300-310.
28 Nowicki PT, Caniano DA, Hammond S, Giannone PJ, Besner GE, Reber KM et al. Endothelial nitric oxide synthase in human intestine resected for necrotizing enterocolitis. J Pediatr 2007; 150(1): 40-45.

29 Vannemreddy P, Notarianni C, Yanamandra K, Napper D, Bocchini J. Is an endothelial nitric oxide synthase gene mutation a risk factor in the origin of intraventricular hemorrhage? Neurosurg Focus 2010; 28(1): E11.

30 Montgomery AM, Bazzy-Asaad A, Asnes JD, Bizzarro MJ, Ehrenkranz RA, Weismann CG. Biochemical screening for pulmonary hypertension in preterm infants with bronchopulmonary dysplasia. Neonatology 2016; 109(3): 190-194.

31 Tanner K, Sabrine N, Wren C. Cardiovascular malformations among preterm infants. Pediatrics 2005; 116(6): e833-e838.

32 Lammers A, Hager A, Eicken A, Lange R, Hauser M, Hess J. Need for closure of secundum atrial septal defect in infancy. J Thorac Cardiovasc Surg 2005; 129(6): 1353-1357.

33 Thomas VC, Vincent R, Raviele A, Diehl H, Qian H, Kim D. Transcatheter closure of secundum atrial septal defect in infants less than 12 months of age improves symptoms of chronic lung disease. Congenit Heart Dis 2012; 7(3): 204-211. 